\title{
DARK MATTER PARTICLES IN THE GALACTIC HALO
}

\author{
R. Bernabei \\ Dip. di Fisica, Università di Roma "Tor Vergata" \\ and INFN-Tor Vergata, I-00133 Rome, Italy
}

July 5,2021

\begin{abstract}
The DAMA/LIBRA-phase1 and the former DAMA/NaI data (cumulative exposure 1.33 ton $\times$ yr, corresponding to 14 annual cycles) give evidence at $9.3 \sigma$ C.L. for the presence of Dark Matter (DM) particles in the galactic halo, on the basis of the exploited model independent DM annual modulation signature by using highly radio-pure $\mathrm{NaI}(\mathrm{Tl})$ target. Results and comparisons will be shortly addressed as well as perspectives of the presently running DAMA/LIBRA-phase2. Finally, some arguments arisen in the discussion section of this workshop are mentioned in the Appendix.
\end{abstract}

\section{Introduction}

About 80 years of experimental observations and theoretical arguments have pointed out that a large fraction of the Universe is composed by Dark Matter particles 1 .

The presently running DAMA/LIBRA $(\simeq 250 \mathrm{~kg}$ of full sensitive targetmass) [1, 2, 3, 4, 5, 6, 7, 8, 9] experiment, as well as the former DAMA/NaI

\footnotetext{
${ }^{1}$ For completeness, it is worth recalling that some efforts to find alternative explanations to Dark Matter have been proposed such as MOdified Gravity Theory (MOG) and MOdified Newtonian Dynamics (MOND); they hypothesize that the theory of gravity is incomplete and that a new gravitational theory might explain the experimental observations. MOND modifies the law of motion for very small accelerations, while MOG modifies the Einstein's theory of gravitation to account for an hypothetical fifth fundamental force in addition to the gravitational, electromagnetic, strong and weak ones. However, e.g.: i) there is no general underlying principle; ii) they are generally unable to account for all small and large scale observations; iii) they fail to reproduce accurately the Bullet Cluster; iv) generally they require some amount of DM particles as seeds for the structure formation.
} 
( $\simeq 100 \mathrm{~kg}$ of full sensitive target-mass) [10, 11, 12, 13, 14, 15, 16], has the main aim to investigate the presence of DM particles in the galactic halo by exploiting the model independent DM annual modulation signature (originally suggested in Ref. [17]).

As a consequence of the Earth's revolution around the Sun, which is moving in the Galaxy with respect to the Local Standard of Rest towards the star Vega near the constellation of Hercules, the Earth should be crossed by a larger flux of DM particles around $\simeq 2$ June and by a smaller one around $\simeq 2$ December. In the former case the Earth orbital velocity is summed to the one of the solar system with respect to the Galaxy, while in the latter the two velocities are subtracted 2 . This DM annual modulation signature is very distinctive since the effect induced by DM particles must simultaneously satisfy all the following requirements: the rate must contain a component modulated according to a cosine function (1) with one year period (2) and a phase that peaks roughly $\simeq 2$ June (3); this modulation must only be found in a well-defined low energy range, where DM particle induced events can be present (4); it must apply only to those events in which just one detector of many (9 in DAMA/NaI and 25 in DAMA/LIBRA) actually "fires" (single-hit events), since the DM particle multi-interaction probability is negligible (5); the modulation amplitude in the region of maximal sensitivity must be $\simeq 7 \%$ for usually adopted halo distributions (6), but it can be larger (even up to $\simeq 30 \%$ ) in case of some possible scenarios such as e.g. those in Ref. [18, 19]. Thus, this signature is model independent and very effective; moreover, the developed highly radio-pure $\mathrm{NaI}(\mathrm{Tl})$ target-detectors [1] and the adopted procedures assure sensitivity to a wide range of DM candidates (both inducing nuclear recoils and/or electromagnetic radiation), interaction types and astrophysical scenarios.

In particular, the experimental observable in DAMA experiments is the modulated component of the signal in $\mathrm{NaI}(\mathrm{Tl})$ target and not the constant part of it as in other approaches as those by CDMS, Xenon, etc., where in addition e.g.: i) different target materials are used; ii) the sensitivity is mainly restricted to candidates inducing just nuclear recoils; iii) many (by the fact largely uncertain) selections/subtractions of detectors and of data and (highly uncertain) extrapolations of detectors' features are applied.

The DM annual modulation signature might be mimicked only by systematic effects or side reactions able to account for the whole observed

\footnotetext{
${ }^{2}$ Thus, the DM annual modulation signature has a different origin and peculiarities than the seasons on the Earth and than effects correlated with seasons (consider the expected value of the phase as well as other requirements listed below).
} 
modulation amplitude and to simultaneously satisfy all the requirements given above. No one is available or suggested by anyone over more than a decade [1, 2, 15, 6, 8, 13, 9].

It is also worth noting that the DM annual modulation signature acts itself as a strong background reduction as pointed out since the early paper by Ref. [17, and especially when all the above peculiarities can be experimentally verified in suitable dedicated set-ups as it is the case of the DAMA experiments.

\section{The DAMA results}

The total exposure of DAMA/LIBRA-phase1 is: 1.04 ton $\times$ yr in seven annual cycles; when including also that of the first generation DAMA/NaI experiment it is 1.33 ton $\times$ yr, corresponding to 14 annual cycles. The variance of the cosine during the DAMA/LIBRA-phase1 data taking is 0.518 , showing that the set-up has been operational evenly throughout the years [2, 6].

Many independent data analyses have been carried out [2, 6] and all of them confirm the presence of a peculiar annual modulation in the single-hit scintillation events in the $2-6 \mathrm{keV}$ energy interval, which - in agreement with the requirements of the DM signature - is absent in other parts of the energy spectrum and in the multiple-hit scintillation events in the same 2-6 $\mathrm{keV}$ energy interval (this latter condition correspond to have "switched off the beam" of DM particles). All the analyses and details can be found in the literature given above. In particular, Fig. 1 shows the time behaviour of the experimental residual rates of the single-hit scintillation events for DAMA/NaI [13] and DAMA/LIBRA-phase1 [2, 6] cumulatively in the (26) $\mathrm{keV}$ energy interval. The data points present the experimental errors as vertical bars and the associated time bin width as horizontal bars. The superimposed curve is the cosinusoidal function $A \cos \omega\left(t-t_{0}\right)$ with a pe$\operatorname{riod} T=\frac{2 \pi}{\omega}=1 \mathrm{yr}$, a phase $t_{0}=152.5$ day (June $2^{\text {nd }}$ ) and modulation amplitude, $A$, equal to the central value obtained by best fit on the data points. The dashed vertical lines correspond to the maximum expected for the DM signal, while the dotted vertical lines correspond to the expected minimum. The major upgrades are also pointed out.

In order to continuously monitor the running conditions, several pieces of information are acquired with the production data and quantitatively analysed. In particular, all the time behaviours of the running parameters, acquired with the production data, have been investigated: the modulation amplitudes obtained for each annual cycle when fitting the time behaviours 


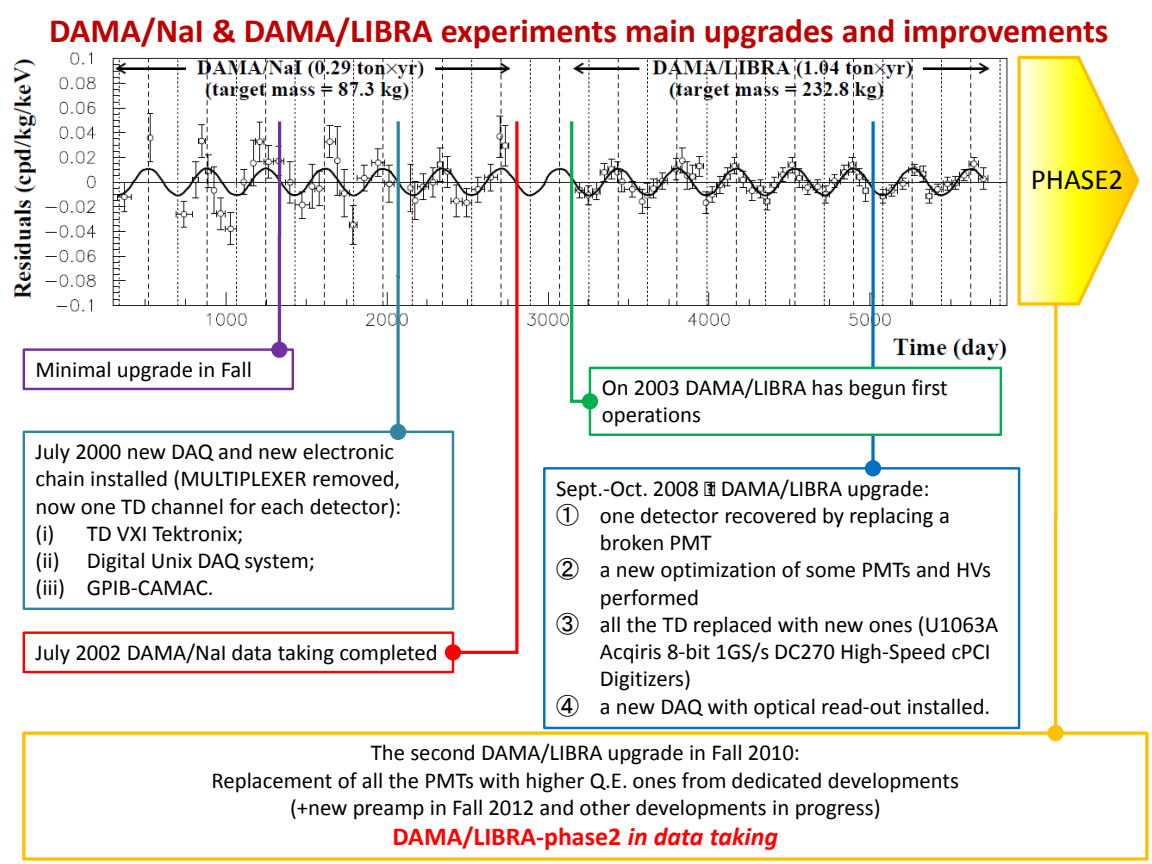

Figure 1: Experimental residual rate of the single-hit scintillation events measured by DAMA/NaI and DAMA/LIBRA-phase1 in the (2-6) keV energy interval as a function of the time. The data points present the experimental errors as vertical bars and the associated time bin width as horizontal bars; see text. As always in DAMA results, the given rate is already corrected for the overall efficiency. The major upgrades of the experiment are also pointed out.

of the parameters including a cosine modulation with the same phase and period as for DM particles are well compatible with zero. In particular, no modulation has been found in any possible source of systematics or side reactions; thus, cautious upper limits (90\% C.L.) on possible contributions to the DAMA/LIBRA measured modulation amplitude have been derived (see e.g. 2]). It is worth noting that they do not quantitatively account for the measured modulation amplitudes, and are not able to simultaneously satisfy all the many requirements of the signature. Similar analyses have also been carried out for the DAMA/NaI data 13 .

No other experimental result has been verified over so long time so accurately and with various significant upgrades of the set-ups.

For completeness I mention that sometimes naive statements were put forwards as the fact that in nature several phenomena may show some kind 
Table 1: Summary of the contributions to the total neutron flux at LNGS; the value, the relative modulation amplitude, and the phase of each component is reported. It is also reported the counting rate in DAMA/LIBRA for single-hit events, in the $(2-6) \mathrm{keV}$ energy region induced by neutrons, muons and solar neutrinos, detailed for each component. The modulation amplitudes, $A_{k}$, are reported as well, while the last column shows the relative contribution to the annual modulation amplitude observed by DAMA, $S_{m}^{e x p} \simeq 0.0112 \mathrm{cpd} / \mathrm{kg} / \mathrm{keV}[2$. As can be seen, they are all negligible and they cannot give any significant contribution to the observed modulation amplitude. In addition, neutrons, muons and solar neutrinos are not a competing background when the DM annual modulation signature is investigated since in no case they can mimic this signature. For details see Ref. [9] and references therein.

\begin{tabular}{|c|c|c|c|c|c|c|c|}
\hline & Source & $\begin{array}{c}\Phi_{0, k}^{(n)} \\
\text { (neutrons } \mathrm{cm}^{-2} \mathrm{~s}^{-1} \text { ) }\end{array}$ & $\eta_{k}$ & $t_{k}$ & $\begin{array}{c}R_{0, k} \\
\text { (cpd/kg/keV) }\end{array}$ & $\begin{array}{c}A_{k}=R_{0, k} \eta_{k} \\
(\mathrm{cpd} / \mathrm{kg} / \mathrm{keV})\end{array}$ & $A_{k} / S_{m}^{\exp }$ \\
\hline \multirow[b]{2}{*}{$\begin{array}{l}\text { SLOW } \\
\text { neutrons }\end{array}$} & $\begin{array}{l}\text { thermal n } \\
\left(10^{-2}-10^{-1} \mathrm{eV}\right)\end{array}$ & $1.08 \times 10^{-6}$ & $\begin{array}{c}\simeq \simeq 0 \\
\text { however } \ll 0.1\end{array}$ & - & $<8 \times 10^{-6}$ & $\ll 8 \times 10^{-7}$ & $\ll 7 \times 10^{-5}$ \\
\hline & $\begin{array}{l}\text { epithermal n } \\
(\mathrm{eV}-\mathrm{keV})\end{array}$ & $2 \times 10^{-6}$ & $\begin{array}{c}\simeq 0 \\
\text { however } \ll 0.1\end{array}$ & - & $<3 \times 10^{-3}$ & $\ll 3 \times 10^{-4}$ & $\ll 0.03$ \\
\hline \multirow{6}{*}{$\begin{array}{l}\text { FAST } \\
\text { neutrons }\end{array}$} & $\begin{array}{l}\text { fission, }(\alpha, n) \rightarrow \mathrm{n} \\
(1-10 \mathrm{MeV})\end{array}$ & $\simeq 0.9 \times 10^{-7}$ & $\begin{array}{c}\simeq 0 \\
\text { however } \ll 0.1\end{array}$ & - & $<6 \times 10^{-4}$ & $\ll 6 \times 10^{-5}$ & $\ll 5 \times 10^{-3}$ \\
\hline & $\begin{array}{l}\mu \rightarrow \mathrm{n} \text { from rock } \\
(>10 \mathrm{MeV})\end{array}$ & $\simeq 3 \times 10^{-9}$ & 0.0129 & $\begin{array}{l}\text { end of } \\
\text { June }\end{array}$ & $\ll 7 \times 10^{-4}$ & $\ll 9 \times 10^{-6}$ & $\ll 8 \times 10^{-4}$ \\
\hline & $\begin{array}{l}\mu \rightarrow \mathrm{n} \text { from } \mathrm{Pb} \text { shield } \\
(>10 \mathrm{MeV})\end{array}$ & $\simeq 6 \times 10^{-9}$ & 0.0129 & $\begin{array}{l}\text { end of } \\
\text { June }\end{array}$ & $\ll 1.4 \times 10^{-3}$ & $\ll 2 \times 10^{-5}$ & $\ll 1.6 \times 10^{-3}$ \\
\hline & $\begin{array}{l}\nu \rightarrow \mathrm{n} \\
(\text { few } \mathrm{MeV})\end{array}$ & $\simeq 3 \times 10^{-10}$ & $0.03342^{*}$ & Jan. $4 \mathrm{th}^{*}$ & $\ll 7 \times 10^{-5}$ & $\ll 2 \times 10^{-6}$ & $\ll 2 \times 10^{-4}$ \\
\hline & direct $\mu$ & $\Phi_{0}^{(\mu)} \simeq 20 \mu \mathrm{m}^{-2} \mathrm{~d}^{-1}$ & 0.0129 & $\begin{array}{l}\text { end of } \\
\text { June }\end{array}$ & $\simeq 10^{-7}$ & $\simeq 10^{-9}$ & $\simeq 10^{-7}$ \\
\hline & direct $\nu$ & $\Phi_{0}^{(\nu)} \simeq 6 \times 10^{10} \nu \mathrm{cm}^{-2} \mathrm{~s}^{-1}$ & $0.03342^{*}$ & Jan. $4 \mathrm{th}^{*}$ & $\simeq 10^{-5}$ & $3 \times 10^{-7}$ & $3 \times 10^{-5}$ \\
\hline
\end{tabular}

* The annual modulation of solar neutrino is due to the different Sun-Earth distance along the year; so the relative modulation amplitude is twice the eccentricity of the Earth orbit and the phase is given by the perihelion.

of periodicity. The point is whether they could mimic the annual modulation signature in DAMA/LIBRA (and former DAMA/NaI), i.e. whether they could quantitatively account for the observed modulation amplitude and also simultaneously satisfy all the requirements of the DM annual modulation signature. The same is also for side reactions. This has already been deeply investigated in Ref. [1, 2] and references therein; the arguments and the quantitative conclusions, presented there, also apply to the entire DAMA/LIBRA-phase1 data. Additional arguments can be found in Ref. [5, 6, 8, 9]. In particular, Ref. [9] further outlines in a simple and intuitive way why neutrons (of whatever origin), muons and solar neutrinos cannot give any significant contribution to the DAMA annual modulation results and - in addition - can never mimic the DM annual modulation 
signature since some of its specific requirements fail. Table 1 summarizes the safety upper limits on the contributions (if any) to the observed modulation amplitude due to the total neutron flux at LNGS, either from $(\alpha, \mathrm{n})$ reactions, from fissions and from muons' and solar-neutrinos' interactions in the rocks and in the lead around the experimental set-up; the direct contributions of muons and solar neutrinos are also reported there. As seen in Table 1, they are all negligible and they cannot give any significant contribution to the observed modulation amplitude; in addition, neutrons, muons and solar neutrinos are not a competing background when the DM annual modulation signature is investigated since they cannot mimic this signature. For details see Ref. [9] and references therein.

In conclusion, DAMA gives a model-independent evidence - at $9.3 \sigma$ C.L. over 14 independent annual cycles - for the presence of DM particles in the galactic halo.

\subsection{On comparisons}

No direct model independent comparison is possible in the field when different target materials and/or approaches are used; the same is for the strongly model dependent indirect searches 3 .

In order to perform corollary investigations on the nature of the DM particles, model-dependent analyses are necessary 4 . Thus, many theoretical and experimental parameters and models are possible (see e.g. in [2, 6, 22, 23]) and many hypotheses must also be exploited, while specific experimental and theoretical assumptions are generally adopted in the field

\footnotetext{
${ }^{3}$ It should be noted that the rising behaviour of the positron flux reported in Ref. 20. 21 does not give any intrinsic evidence for production due to DM annihilation; this may arise only when a particular model of the competing background is assumed as e.g. the GALPROP code. But other more complete models exist which do not support any significant excess evidence. Moreover, an interpretation in terms of DM particle annihilation would require the assumption of: i) a very large boost factor $(\sim 400)$ of the density; ii) to boost the annihilation cross section through an assumed new interaction type; iii) to adjust the propagation parameters; iv) to consider extra-source (subhalos, IMBHs); v) to consider only a leptophilic candidate to justify the absence of any excess in the antiproton spectrum. Finally, other well known sources can account for a similar positron fraction: pulsars, supernova explosions near the Earth, SNR.

${ }^{4}$ For completeness, it is worth recalling that it does not exist any approach to investigate the nature of the candidate in the direct and indirect DM searches, which can offer this information independently on assumed astrophysical, nuclear and particle Physics scenarios. On the other hand, searches for new particles beyond the Standard Model of particle Physics at accelerators cannot credit by themselves that a certain particle is in the halo as a solution or the only solution for DM particles, and - in addition - DM candidates and scenarios (even for the neutralino) exist which cannot be investigated there.
} 
assuming a single arbitrary scenario without accounting neither for existing uncertainties nor for alternative possible scenarios, interaction types, etc.

The obtained DAMA 9.3 $\sigma$ C.L. model independent evidence is compatible with a wide set of scenarios regarding the nature of the DM candidate and related astrophysical, nuclear and particle Physics. For examples some scenarios and parameters are discussed e.g. in Ref. [10, 11, 13, 2, 6, 22, 23]. Further large literature is available on the topics (see for example in the bibliography of Ref. [6]). By the fact, both the negative results and all the possible positive hints are largely compatible with the DAMA modelindependent DM annual modulation results in various scenarios considering also the existing experimental and theoretical uncertainties; the same holds for the strongly model dependent indirect approaches.

It is also worthwhile to further recall that these DAMA experiments are not only sensitive to DM particles with spin-independent coupling inducing just nuclear recoils, but also to other couplings and to other DM candidates as those giving rise to part or all the signal in electromagnetic form. Finally, scenarios exist in which other kind of targets/approaches are disfavoured or even blind.

\section{DAMA/LIBRA-phase2 and perspectives}

An important upgrade has started at end of 2010 replacing all the PMTs with new ones having higher Quantum Efficiency; details on the developments and on the reached performances in the operative conditions are reported in Ref. [4]. They have allowed us to lower the software energy threshold of the experiment to $1 \mathrm{keV}$ and to improve also other features as e.g. the energy resolution [4].

Since the fulfillment of this upgrade and after some optimization periods, DAMA/LIBRA-phase2 is continuously running in order e.g.: (1) to increase the experimental sensitivity thanks to the lower software energy threshold; (2) to improve the corollary investigation on the nature of the DM particle and related astrophysical, nuclear and particle physics arguments; (3) to investigate other signal features and second order effects. This requires long and dedicated work for reliable collection and analysis of very large exposures.

In the future DAMA/LIBRA will also continue its study on several other rare processes as also the former DAMA/NaI apparatus did.

Finally, further future improvements of the DAMA/LIBRA set-up to increase the sensitivity (possible DAMA/LIBRA-phase3) and the developments towards the possible DAMA/1ton (1 ton full sensitive mass on the 
contrary of other kind of detectors), we proposed in 1996, are considered at some extent. For the first case developments of new further radiopurer PMTs with high quantum efficiency are starting, while in the second case it would be necessary to overcome the present problems regarding: i) the supplying, selection and purifications of a large number of high quality $\mathrm{NaI}$ and, mainly, TlI powders; ii) the availability of equipments and competence for reliable measurements of small trace contaminants in ppt or lower region; iii) the creation of updated protocols for growing, handling and maintaining the crystals; iv) the availability of large Kyropoulos equipments with suitable platinum crucibles; v) etc.. At present, due to the change of rules for provisions of strategical materials, the large costs and the lost of some equipments and competence also at industry level, a satisfactory development appears quite difficult.

\section{Conclusions}

The data of DAMA/LIBRA-phase1 have further confirmed the presence of a peculiar annual modulation of the single-hit events in the $(2-6) \mathrm{keV}$ energy region satisfying all the many requirements of the DM annual modulation signature; the cumulative exposure by the former DAMA/NaI and DAMA/LIBRA-phase1 is 1.33 ton $\times$ yr (orders of magnitude larger than those typically released in the field).

As required by the DM annual modulation signature: 1) the single-hit events show a clear cosine-like modulation as expected for the DM signal; $2)$ the measured period is equal to $(0.998 \pm 0.002)$ yr well compatible with the $1 \mathrm{yr}$ period as expected for the DM signal; 3) the measured phase $(144 \pm 7)$ days is compatible with $\simeq 152.5$ days as expected for the DM signal; 4) the modulation is present only in the low energy (2-6) keV interval and not in other higher energy regions, consistently with expectation for the DM signal; 5) the modulation is present only in the single-hit events, while it is absent in the multiple-hit ones as expected for the DM signal; 6 ) the measured modulation amplitude in $\mathrm{NaI}(\mathrm{Tl})$ of the single-hit events in the $(2-6) \mathrm{keV}$ energy interval is: $(0.0112 \pm 0.0012) \mathrm{cpd} / \mathrm{kg} / \mathrm{keV}(9.3 \sigma$ C.L.). No systematic or side processes able to simultaneously satisfy all the many peculiarities of the signature and to account for the whole measured modulation amplitude is available.

DAMA/LIBRA-phase2 is continuously running in its new configuration with a lower software energy threshold aiming to improve the knowledge on corollary aspects regarding the signal and on second order effects as discussed e.g. in Ref. 6, 8]. 
Few comments on model-dependent comparisons have also been addressed here.

\section{Acknowledgments}

It is a pleasure to thank all my DAMA collaborators who effectively dedicated their efforts to this experimental activity and the colleagues in this Workshop for the interesting topics we have discussed, for the question section, and for the pleasant scientific environment.

\section{Appendix: Questions \& Answers}

This section shortly summarizes some of the topics extensively discussed at the Workshop, where the time dedicated to discussions and the interest in deeply understanding the topics were rather large.

Question 1: may you comment about the ratio of the measured dark matter particles modulation amplitude to the total signal: the $S_{m} / S_{0}$ ratio?

Answer 1: the measured counting rate in the cumulative energy spectrum is about $1 \mathrm{cpd} / \mathrm{kg} / \mathrm{keV}$ in the lowest energy bins; this is the sum of the background contribution and of the constant part of the signal $\mathrm{S}_{0}$. As discussed e.g. in TAUP2011 [24], the background in the 2-4 keV energy region is estimated to be not lower than about $0.75 \mathrm{cpd} / \mathrm{kg} / \mathrm{keV}$; this gives an upper limit on $\mathrm{S}_{0}$ of about $0.25 \mathrm{cpd} / \mathrm{kg} / \mathrm{keV}$. Thus, the $\mathrm{S}_{m} / \mathrm{S}_{0}$ ratio is equal or larger than about $0.01 / 0.25 \simeq 4 \%$.

Question 2: may you comment on the quenching factors, on their dependence on the type of the particles, and on some typical examples of extreme properties?

Answer 2: The quenching factor values play a role only when corollary model-dependent analyses for DM candidates inducing just nuclear recoils are carried out, in order to derive the energy scale in terms of nuclear recoil energy.

As is widely known, the quenching factor is a specific property of the employed detector and not a general quantity universal for a given material. For example, in liquid noble-gas detectors, it depends - among others - on the level of trace contaminants which can vary in time and from one liquefaction process to another, on the cryogenic microscopic conditions, etc.. In bolometers it depends for instance on specific properties, 
trace contaminants, cryogenic conditions, etc. of each specific detector, while generally it is assumed exactly equal to unity (the maximum possible value). The quenching factors in scintillators depend, for example, on the dopant concentration, on the growing method/procedures, on residual trace contaminants, etc., and are expected to be energy dependent. Thus, all these aspects are already by themselves relevant sources of uncertainties when interpreting whatever result in terms of DM candidates inducing just nuclear recoils. Similar arguments have been addressed e.g. in Ref. $[2,3,13,15,25$.

Question 3: May you comment under which extreme conditions your experiment is successful and comment what can at most the experiment which does not fulfil one of the conditions or more than one of them at most can "see"?

Answer 3: The full description and potentiality of the DAMA/LIBRA set-up have been discussed in details in Refs. 1, 2, 4, and references therein. Obviously all the set-up specific features and adopted procedures contribute to the possibility to point out the signal through the model independent DM annual modulation signature. The absence/difference of one of them would limit whatever else result.

\section{Question 4: May you comment about muons?}

Answer 4: An extensive discussion on this topics can be found in the dedicated Ref. [5, 9], where its has been quantitatively demonstrated (see also Table 1 in this paper) that - for many reasons (and just one would suffice) - muons cannot play (directly or indirectly) any role in the DAMA annual modulation effect.

Question 5: May you comment about neutrinos?

Answer 5: The contribution from solar, atmospheric, .. neutrinos is obviously negligible; a quantitative discussion can be found in Ref. [9] (see also Table 1 in this paper).

Question 6: May you comment about the operating temperature of your measuring apparatus?

Answer 6: The DAMA set-ups operate at environmental temperature maintained stable by suitable and redundant air-conditioning system (2 independent devices for redundancy); moreover, the $\mathrm{Cu}$ housings of the detectors are in direct contact with the multi-ton metallic shield, thus there 
is a huge heat capacity $\left(\sim 10^{6} \mathrm{cal} /{ }^{0} \mathrm{C}\right)$. In addition, the operating temperature of the detectors is continuously monitored and analysed as the production data. A discussion on temperature in operating condition can be found e.g. in Ref. [2, 6].

Question 7: May you comment about the Snowmass plots and its meaning?

Answer 7: The recent plot from Snowmass and that in Ref. [26] about the "status of the Dark Matter search" do not point out at all the real status of Dark Matter searches since e.g.: i) Dark Matter has wider possibilities than WIMPs inducing just nuclear recoil with spin-independent interaction under single (largely arbitrary) set of assumptions; ii) neither the uncertainties for existing experimental and theoretical aspects nor alternative possible assumptions are accounted for; iii) they do not include possible systematic errors affecting the data (such as e.g. "extrapolations" of energy threshold, of energy resolution and of efficiencies, quenching factors values, convolution with poor energy resolution, correction for non-uniformity of the detector, multiple subtractions/selection of detectors and/or data, assumptions on quantities related to halo model, form factors, scaling laws, etc.); iv) the DAMA implications - even adopting the many arbitrary assumptions considered there - appear incorrect, for example the $S_{0}$ prior is not accounted for, etc., etc.. The perspectives as well appear incorrect/too optimistic.

\section{References}

[1] R. Bernabei et al., Nucl. Instr. and Meth. A 592 (2008) 297.

[2] R. Bernabei et al., Eur. Phys. J. C 56 (2008) 333; Eur. Phys. J. C 67 (2010) 39; Eur. Phys. J. C 73 (2013) 2648.

[3] P. Belli et al., Phys. Rev. D 84 (2011) 055014.

[4] R. Bernabei et al., J. of Instr. 7 (2012) P03009.

[5] R. Bernabei et al., Eur. Phys. J. C 72 (2012) 2064.

[6] R. Bernabei et al., Int. J. of Mod. Phys. A 28 (2013) 1330022.

[7] R. Bernabei et al., Eur. Phys. J. C 62 (2009) 327; Eur. Phys. J. C 72 (2012) 1920; Eur. Phys. J. A 49 (2013) 64.

[8] R. Bernabei et al., Eur. Phys. J. C 74 (2014) 2827. 
[9] R. Bernabei et al., arXiv:1409.3516 in press on Eur. Phys. J. C.

[10] R. Bernabei et al., Phys. Lett. B 389 (1996) 757.

[11] R. Bernabei et al., Phys. Lett. B 424 (1998) 195; Phys. Lett. B 450 (1999) 448; Phys. Rev. D 61 (2000) 023512; Phys. Lett. B 480 (2000) 23; Phys. Lett. B 509 (2001) 197; Eur. Phys. J. C 23 (2002) 61; P. Belli et al., Phys. Rev. D 66 (2002) 043503.

[12] R. Bernabei et al., Il Nuovo Cim. A 112 (1999) 545.

[13] R. Bernabei et al., La Rivista del Nuovo Cimento 26 n.1 (2003) 1-73; Int. J. Mod. Phys. D 13 (2004) 2127.

[14] R. Bernabei et al., Int. J. Mod. Phys. A 21 (2006) 1445; Eur. Phys. J. C 47 (2006) 263; Int. J. Mod. Phys. A 22 (2007) 3155; Phys. Rev. D 77 (2008) 023506; Mod. Phys. Lett. A 23 (2008) 2125.

[15] R. Bernabei et al., Eur. Phys. J. C 53 (2008) 205.

[16] R. Bernabei et al., Phys. Lett. B408 (1997) 439; P. Belli et al., Phys. Lett. B460 (1999) 236; Phys. Rev. C60 (1999) 065501; R. Bernabei et al., Phys. Rev. Lett. 83 (1999) 4918; Il Nuovo Cimento A112 (1999) 1541; Phys. Lett. B 515 (2001) 6; F. Cappella et al., Eur. Phys. J.direct C14 (2002) 1; R. Bernabei et al., Eur. Phys. J. A 23 (2005) 7; Eur. Phys. J. A 24 (2005) 51; Astrop. Phys. 4 (1995) 45; R. Bernabei, in the volume The Identification of Dark Matter, World Sc. Pub. (1997) 574 .

[17] K.A. Drukier et al., Phys. Rev. D 33 (1986) 3495; K. Freese et al., Phys. Rev. D 37 (1988) 3388.

[18] D. Smith and N. Weiner, Phys. Rev. D 64 (2001) 043502; D. TuckerSmith and N. Weiner, Phys. Rev. D 72 (2005) 063509; D. P. Finkbeiner et al., Phys. Rev. D 80 (2009) 115008.

[19] K. Freese et al., Phys. Rev. D 71 (2005) 043516; K. Freese et al., Phys. Rev. Lett. 92 (2004) 11301.

[20] O. Adriani et al., Phys. Rev. Lett. 111 (2013) 081102.

[21] L. Accardo et al. (AMS Coll.), Phys. Rev. Lett. 113 (2014) 121101.

[22] G. Bregar and N.S. Mankoc Borstnik, Phys. Rev. D 80 (2009) 083534.

[23] Q. Wallemacq and J.R. Cudell, arXiv:1411.3178. 
[24] R. Bernabei et al., J. Phys.: Conf. Ser. 203, 012040 (2010), arXiv:0912.4200 http://taup2009.lngs.infn.it/slides/jul3/nozzoli.pdf, talk given by F. Nozzoli].

[25] V. I. Tretyak, Astropart. Phys. 33 (2010) 40.

[26] Review of Particle Properties: http://pdg.lbl.gov/2013/reviews/rpp2013-rev-dark-matter.pdf 